\title{
Collaborative capacity planning and control in subcontracting networks
}

\author{
I.P. Tatsiopoulos \\ National Technical University of Athens, 15780 Zografos, Athens, Greece. \\ Email: itat@central.ntua.gr
}

\begin{abstract}
This study has the objective to apply new Internet technologies and multi-agent modelling in order to support the business process of Production Orders Release and especially its capacity planning and control dimension in subcontracting networks. In a previous publication an object-oriented Orders Release reference model has been defined as a link between production management and shop floor control software in the one-firm environment. This study has the objective to extend this model in the multi-firm extended enterprise and to build a multi-agent based system that takes advantage of the Internet.
\end{abstract}

Keywords

Orders release, Workload control, Extended Enterprise, Capacity Planning.

\section{INTRODUCTION}

Orders Release includes those activities, which must take place before an order defined by a planning system can be released to an execution system. These activities are necessary to control the flow of information and orders passing from the planning system to the execution system and to ensure that the orders released have a reasonable chance of being fully completed (the requested quantity within the agreed time period). According to the above definition, traditional Orders Release forms the link between the production planning system and the shop floor control system (s). In this study, that concerns extended/virtual enterprises, the terms planning system and execution system are recursively meant at all levels of the ISO manufacturing model (enterprise-factory-cell-work centre machine / workplace), see Browne et al. 1991. At the enterprise level of a multi-plant extended enterprise, which is here the main focus, execution systems are the particular factories which are usually considered as black boxes by the planning system of the extended enterprise.

In terms of production management software packages, the Orders Release process has a central role that defines the whole manufacturing order cycle both from a function and a data point of view. There is currently no common architecture of the Orders Release process that would allow different vendors of production management software to cooperate. There is currently no integrated theory of which 
objects, data structures and processes are required in an application module. Examples of such generic models, like the COSIMA effort for shop floor control (Browne et al. 1991) concentrate on parts of the problem, i.e. the function view of mainly time-based problems on the shop floor. The COSIMA architecture is based on three main building blocks, the Scheduler, the Dispatcher and the Monitor, which are recursively repeated at the manufacturing cell control level and at the factory coordination level. In the proposed here architecture there is a significant extension of the dispatcher function, which takes the name of the Releaser in order to reflect its broader role. The COSIMA definition of the Dispatcher as a real-time Scheduler is most appropriate at the decision levels close to the factory floor (cell and work centre control). At more distant decision levels (factory coordination and enterprise logistics management), the dispatcher should assume a wider role including all actions associated to hierarchical Orders Release. This is done in the sense of the basic management function of delegation at all decision levels of the management hierarchy.

In a previous publication (Tatsiopoulos 1997) an object-oriented Orders Release reference model has been defined as a link between production management and shop floor control software in the one-firm environment. This study has the objective to extend the capacity planning and control or workload control dimensions of this model in the multi-firm extended enterprise and to build a multiagent based system that takes advantage of the Internet.

\section{LITERATURE REVIEW}

The manufacturing enterprises of the $21^{\text {st }}$ century will have to survive in an environment where markets are frequently shifting, new technologies are continuously emerging and competition becomes rough-and-tumble. Therefore the next generation manufacturing systems will have to be more market-response oriented, while still focusing on cost and quality. Shen \& Norrie (1999), believe that state of the art manufacturing systems of the new century will need to satisfy the following fundamental requirements, such as enterprise integration, distributed organisation, heterogeneous environments, interoperability, open structure, cooperation, agility, scalability and fault tolerance.

Techniques and concepts from Artificial Intelligence (AI) have already been used in the domain of intelligent manufacturing under the umbrella of a newly emerged scientific domain, this of Distributed Artificial Intelligence (DAI). The issue of flexible allocation of tasks to multiple problem solvers (nodes) received attention early on. Davis and Smith's (1983) work resulted in the well-known Contract Net Protocol (see also Sandholm 1997 and Jennings et al 1998). In this protocol, agents can dynamically take two roles: manager or contractor. Given a task to perform, an agent first determines whether it can break it into subtasks that could be performed concurrently. It employs the Contract Net protocol to announce the tasks that could be transferred, and requests bids from nodes that could perform any of these tasks. A node that receives a task announcement replies with a bid for that task, indicating how well it thinks it can perform the task. The contractor collects the bids and awards the task to the best bidder. Parunak (1987) describes the 
YAMS (Yet Another Manufacturing System) system, which applies the Contract Net Protocol to manufacturing control in a multi-plant environment. YAMS adopts a multi-agent approach, where each factory and factory component is represented as an agent. Each agent has a collection of plans, representing its capabilities. The contract net protocol allows tasks (i.e. production orders) to be delegated to individual factories, and from individual factories to FMSs, and then to individual work cells. Sadeh and Fox (1989) describe CORTES as a two-agent system responsible for solving scheduling problems using micro-opportunistic techniques. Each agent is responsible for scheduling a set of jobs and for monitoring a set of resources. Resources are shared in the architecture and coordinated by a monitor. Saad et al (1995) describes a Production reservation approach using a bidding mechanism based on the Contract Net protocol to generate the production plan and schedule. Chavez and Maes (1996) describe a simple "electronic market place" called Kasbah that realises the marketplace by creating "buying" and "selling" agents for each good to be purchased or sold respectively. Commercial transactions then take place by the interactions of these agents. Sadeh et al (2001) provides an overview of MASCOT, a reconfigurable, multi-level, agent-based planning and scheduling architecture aimed at supporting production and transportation across supply chains that are dynamically setup. Petersen et al (2001) describe how virtual enterprises can be modelled using the AGORA multi-agent architecture, designed for modelling and supporting cooperative work among distributed entities. The model consists of a structure of AGORAs and agents, where AGORAs are facilitators of cooperative work for agents and the agents represent the partners of the VE, the cooperative mechanisms and the service providers. Finally, Wortmann and Szirbik (2001) present a review of the current trends in IT interorganizational integration. It is argued that the conceptual, structural and technological changes in the enterprise-to-enterprise interaction are producing a paradigm shift towards the component and agent technologies. Nevertheless, one should not ignore the key problems that arise in agent-based software engineering such as synchronisation, security, payment for services, crash recovery and inconsistencies in programme specifications (Genesereth 1992). The main features of the agent paradigm along with a short review of the framework, tools and other technology related aspects is presented in the following section.

\section{E-RELEASER FRAMEWORK}

\section{Methodology}

Agent systems are described from the perspective of two research communities: Artificial Intelligence (AI) and Computer Systems (CS). This study adopts the CS approach, where an agent is considered to be a process, a piece of running code with data and code (Tanenbaum \& M. van Steen 2002). Agents are processes that are autonomous and pro-active (capable of making "their own" decisions when they like), interacting, and may be mobile. Agents are often related to objects, where the latter are generally considered to be passive (Jennings \& Wooldridge 1998). In other words, an object needs to be invoked in order to perform a function, and performs 
only during an invocation. Agents, on the other hand, receive messages and autonomously decide if, when, and how to (re)act.

Nevertheless, the requirements analysis phase of building agent-based systems in an Internet environment has to follow the existing object-oriented $(\mathrm{OO})$ analysis tools and techniques, in order to provide the blueprints of a so-called WIS (Web Information System), see Wang (2001). Out of the various OO representation methods (UML by Booch et al (1999), OOA, etc) we have chosen in this study the OOA (Object Orientd Analysis) method of Coad and Yourdon (1991).

The E-RELEASER reference model is built using object orientation for multiagents modelling and consists of four major elements according to OOA:

1. Subjects (overview of a larger OOA model. Subjects are used to guide a reader through a large model)

2. Objects definition and structures (some of the objects have the necessary autonomy to be characterized as agents)

3. Attributes (data view)

4. Services and message connections layer (object/agent behaviour)

\section{Subject areas of $\mathbb{E}$-Releaser}

The subjects view presents the overall architecture of the reference model using a building block diagram that more ore less corresponds to the application module structure that include all objects and agents. The Orders Release mechanism of the E-RELEASER has to solve the following problems in the extended/virtual enterprise environment:

- Partner selection and technical skills availability.

- Order documentation and the related problem of Bills of Material (BOM) and

Process Plans activation.

- Capacity availability and workload control.

- Material availability and material disposition.

These problems can be grouped and solved by three separate subject areas: Partner and Order Manager (Partner selection, Order Documentation), Material Manager (material availability, material disposition) and Capacity Manager (capacity availability, workload control). This leads to a generic system architecture with three basic subject areas that include multi-agents and their assembly structure as depicted in Figure 1.

According to this Figure, the traditional one-firm Enterprise accepts Customer Orders (COs) and releases MRP Planned Production Orders (PPOs) to the Factory (ies), the Factory releases Work Orders (WOs) to the Lines/Cells, the Line/Cell releases (dispatches) Process Batches to the work centres. The M: $\mathrm{N}$ relations between the order types emphasise the lot-sizing decision that exists at all order releasing levels along the material and capacity control decisions.

For the purpose of the E-Releaser in the multi-firm extended enterprise we have to understand and take into consideration the fact that parts across all the above ISO hierarchy of manufacturing resources may be outsourced. This means that either the factory (ies) or some of the line/cells or even some specific processes (workstation level) may belong to a different manufacturing partner belonging to the extended 
enterprise. This partner will produce either integrated assemblies or components of the final product or even specific processes on subcontracting basis. In all cases an order has to be released and SKUs (Stock Keeping Units) with related warehouses have to be handled before and after the outsourcing transaction

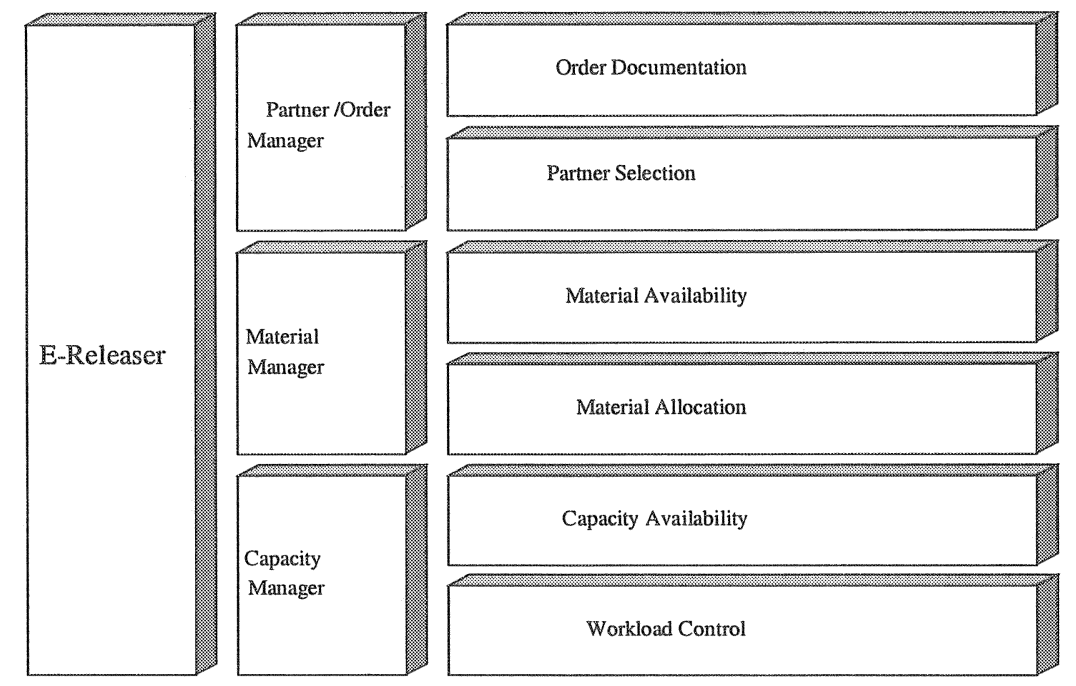

Figure 1-Subject layer of E-Releaser

There follows a description of the discrete modules that are associated to collaborative capacity planning and control

\section{Partmer Selection}

An extended enterprise is characterised by networks of cooperating companies, each one specialised in different production stages. There is usually a primary contractor that collects customer orders and is responsible for transforming them to production orders. The latter are distributed to manufacturing partners who deliver final or semi-final products back to the primary contractor. The partner selection is a time and cost consuming process aiming at the best possible selection from a group of candidate firms. The study of the partner selection process led to the development of an agent that provides information on manufacturing skills and capacities of potential partners. Additionally, it estimates partner performance through performance measurement techniques.

In production terms, the first task in order to assign a production order to a particular partner is to evaluate his manufacturing skills and technical ability to perform the required product components or manufacturing operations. If this test proves to be positive, the next step is to check the gross capacity requirements of the released orders against the planned machine capacity of the candidate partners. This task is performed in cooperation with the agent «capacity manager». 


\section{Capacity Manager}

Capacity availability: The difference between the planning and the execution phase in production systems is that of the planning data versus actual data about capacities and manufacturing lead times. The Orders Release process, being the necessary link between production planning and shop floor execution, has to compare capacity planning data with actual capacity data and make decisions based on the deviations found. In the same sense that the actual material availability is checked, the actual capacity availability connects to the monitoring function in order to find out the actual partner status concerning present personnel, production machinery availability and present capacity load due to running orders (work-inprocess).

Workload control: Workload control based on input/output control methodologies in order to control mean manufacturing lead times. Analysing the orders release process in the industrial practice, it can be observed that not all orders are released to the factory immediately after it becomes theoretically possible to do so. Rather they are retained in a "suspense file", thus being nothing more than a notation in a scheduling book which takes the form of an "order pool" (Irastorza 1974).

Utilisation of this order pool can reduce the level of work-in-process and allow more control over the flow of orders through the supply chain. This is in fact equivalent to cleaning up the supply chain by not allowing excess orders to move into the supply chain. The key to the successful use of the order pool is the availability of a good mechanism to select those orders from the pool that should be released. This mechanism is in fact the Input/ Output Control (IOC) (Kingsman et al. 1989, Bechte 1988, Wiendahl 1987, Bertrand et al. 1991) relying on the aggregate released methodology. The concept of discrete orders release (not timephased) using the Input/Output Control (IOC) methodology is described below:

Whole partner factories performing a single manufacturing operation or a sequence of operations are considered as black box capacity units. The role of the "order pool" is played by the unreleased Production Orders (PPOs) file.

- At the supply chain release level a workload control method has been developed, which is directed rather to the balance of production flow through the supply chain rather than the balance of capacities. This method is characterised by two main principles: Production orders are allowed to remain unreleased for up to a maximum of a few time periods to form a backlog of unreleased orders (pool), with the maximum delay added to the manufacturing time to obtain the lead time.

- Production orders are released periodically in such a way that each partner or indoor work centre and all its downstream partners' work centres are provided with a balanced inflow of work so that their mean released backlogs lengths (actual queue plus released work residing in the upstream partners/work centres) do not exceed their maximum limits.

To solve this problem an aggregate input/output approach can be used relying on the aggregate released backlog instead of actual queues. The aggregate released backlog of a subcontracting partner is defined as the sum of all released work for 
this particular partner, regardless of where it resides, either in the partner's actual queue or in the queue of any other previous (upstream) partner.

The main advantage of the aggregate released backlog as a planning tool is that all the operations of a production order to be released join the released backlogs of the corresponding partners in the order sequence simultaneously at the order release time and stay there until the order is processed and leaves the partner. This means that input to the aggregate released backlog can be easily controlled for all the partners.

The basic tool of our approach for making input control interactive decisions is the backlog length chart (Figure 1). A basic concept of this tool is the Backlog Lineof-Balance (B-LOB). This concept has its origin in the Line-of-Balance (LOB) technique of production control in batch production (Bestwick \& Lockyer 1982). In our case the LOB concept has been combined with the Input/Output Control (IOC) concept and produced the B-LOB technique which is suitable for applying IOC in flow shops. The characteristics of this graphical tool are:

m The bars represent backlog lengths of partners/work centres, i.e. relationships between backlogs and capacities. The backlog lengths are multiples of capacity and they change with capacity even if the backlogs remain the same.

* The chart is not time-phased. The backlog lengths of all the partners are depicted at the same time period. On the contrary the classical load reports show time-phased capacity requirements of just one work centre at a time, so that the overall load situation cannot be grasped at a glance.

- All the operations of a production order are loaded simultaneously at the time period of order entry, so that the inaccurate loading due to the uncertainty of interoperation transit time is avoided.

- The released backlogs of downstream partners/work centres are multiples of the released backlog of the gateway partner/work centre. In the case of flow shops this is analogous to the position of the work centre in the sequence of operations forming a "line-of-balance" for the ideally balanced shop (thick line profile in Figure 2).

- Norms of maximum and minimum backlog lengths are depicted on the chart. Actual performance can then be drawn on the chart, the difference between plan and performance becoming obvious. It is very useful when progressing because it is immediately obvious when corrective action needs to be taken.

Assumed is that the master plan (overall LOB-norm) is fixed by one central point in the manufacturing network, which the prime contractor who has direct contact to the customers. At the moment of an order release to the manufacturing network, there is a contribution to the aggregate released backlog of each partner/work centre, that the order will visit. After operation completion, the amount of work (set-up and process time) is deducted from the released backlog of the respective work centre. Every moment we know the released backlog of every partner/work centre, and by controlling the levels of the released backlogs in each partner/work centre, with the predetermined norms of the line-of-balance, we ensure that the backlog and the flow of work through the shop is smooth and controlled.

By means of the above methodology we can also control the operation lead times and the total manufacturing lead times, moreover to compute and apply more 
reliable delivery times for customer orders. Knowing the capacity of a partner/work centre, in standard labour hours per day, and the predetermined backlog of work for a certain work centre we can compute the mean lead time for the work orders. The mean lead time is equal to the ratio of the backlog of work and the output of work (capacity).

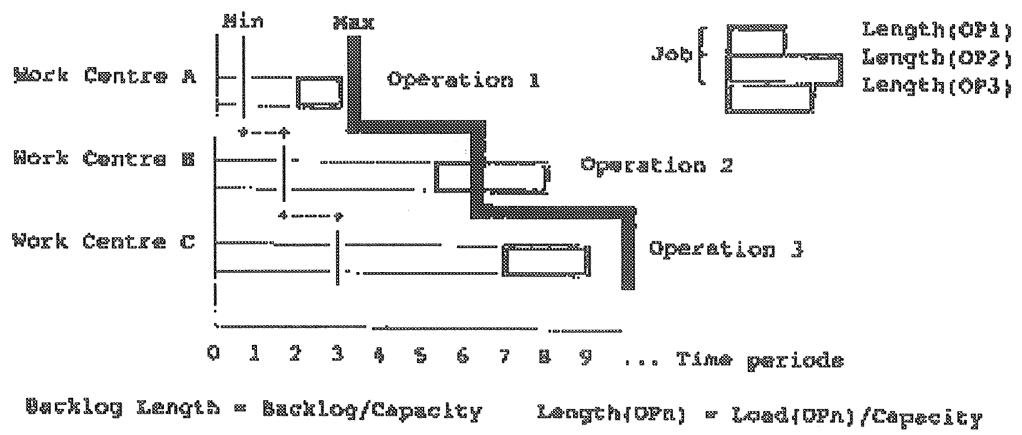

Figure 2 - Backlog length chart and Line-Of-Balance

\section{DESIGN SPECIFICATION}

E-RELEASER is specified as a Web-based Information System (WIS), i.e. an information system (IS) that is based on Web technology and is tightly integrated with conventional IS such as databases and transaction processing systems. Any WIS that has the purpose to support an extended enterprise needs a systems design framework (Davis \& O'Sallivan 1999) significantly enriched in comparison to conventional business data processing design specification methods.

The Davis \& O'Sallivan (1999) framework defines for each business process of the extended enterprise four layers: (1) Interface layer, (2) Communication layer, (3) Service layer and (4) Database layer. A further step for a general model for specifying WIS is taken by Wang (2001), who adopts an object-oriented systems development approach that integrates five basic components: (1) Web sites, (2) business processes, (3) knowledge objects, (4) information infrastructure, and (5) software agents.

In our case the WIS of E-RELEASER facilitates internal activities (intranets) and interaction with partners (extranets). Online activities include partner selection, order release and acceptance, and capacity scheduling. IT infrastructure is based on TCP/IP. Powerful servers link terminals and personal computers at the partners' and factories' locations. A proportion of the WIS is installed behind the enterprise partners' firewalls and is integrated into the partners' systems through creating extranets. The extranets allow the partners to use a service without dial-up to initiate the service.

As shown in Figure 3, the Web site objects in this case are online production order form, online partner inquiry, and online production order tracking inquiry. The 
major business process is Production Orders Release. The objects related to the business process are Manufacturing Partner and Production Order. Triggered objects are Documenting, Material Manager and Capacity Agent. Document objects include Order Docs, Material Slips and Bar Code Labels. The information infrastructure objects are Intranet and Extranet that in turn have Protocol and Firewall objects. In this case, the software agent objects are an intelligent search engine for manufacturing partner selection (Partner Selection Agent) and the Capacity Agent for capacity availability checking.

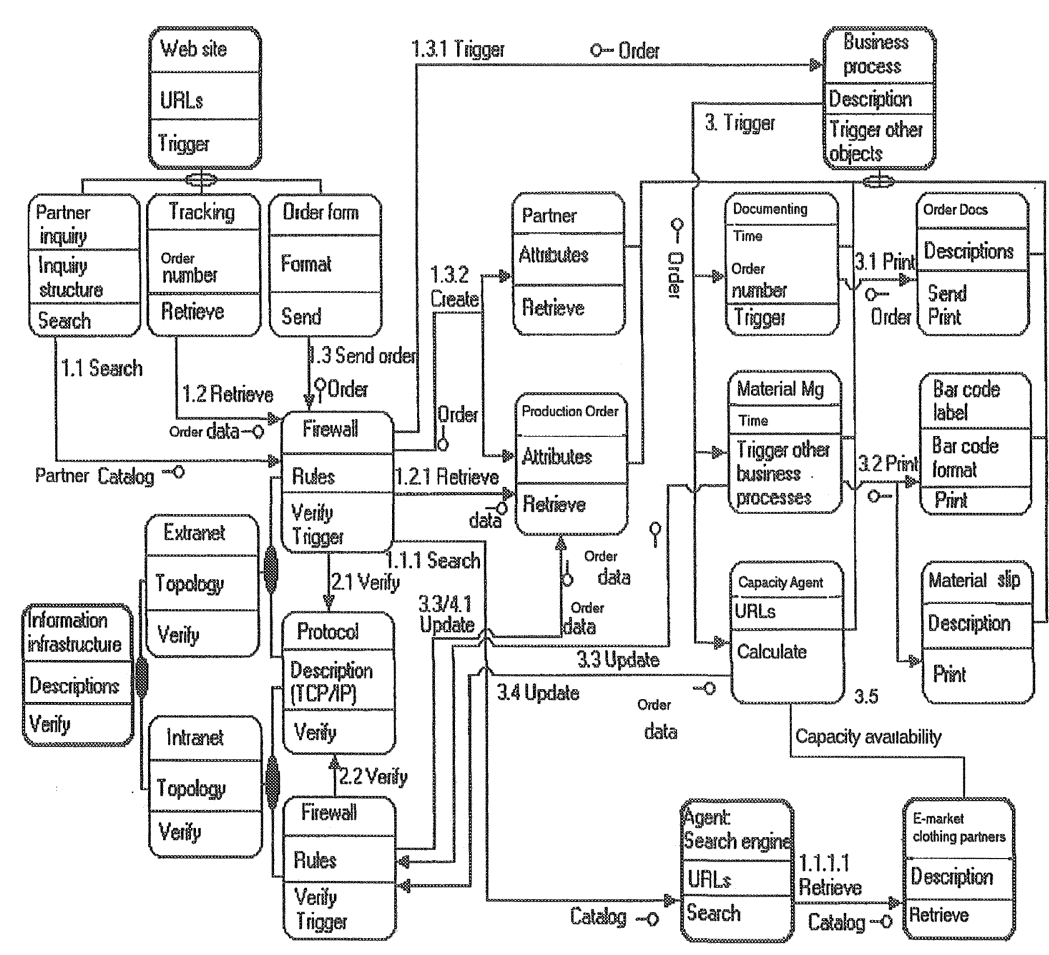

Figure 3 - Design Specification of E-RELEASER Web Information System

The synthesis is started with the Web sites. The message group 1 (Messages 1.1 -1.3 ) is sent by the Web sites, representing the requests for partner inquiry, production order tracking, and online production order entry, respectively. These messages are filtered by extranet firewalls before they trigger business processes and agents. As shown in Figure 3, these messages are relayed after the firewalls. Message 1.1.1 triggers the Partner Selection Agent and retrieves partner information (financial, technical, capacity, performance) from an internet portal and e-market of potential manufacturing partners. Message 1.2.1 retrieves production order tracking information. Message 1.3.1 triggers the business processes of Orders Release. Message 1.3.2 creates the business objects production order and partner. 
Message group 2 (Messages 2.1 and 2.2) represents the verification relationships between the firewalls and other element (s) of the information infrastructure. Message 3 symbolises the response of the business process objects to the online production orders. Messages 3.1 and 3.2 indicate the generation of the business document objects. Messages 3.3 and 3.4 are sent to the enterprise server firewalls in order to update the production order's information which is stored on the server behind the firewalls. Message 3.5 triggers the Capacity Agent, who is responsible for the management, successful retrieval and exporting of the subcontractor capacity data in request, and then downloading and importing the data set into the capacity availability function. After that, the capacity workload function is ready to calculate the subcontractor's potential loading and therefore can determine his ability to undertake the production order in demand.

Note that these messages accentuate the dynamic relationships between the objects, but do not represent particular access paths. As shown above, the synthesis of integration of the categories of object and agent descriptions actually builds the associations between objects and agents by identifying the messages between them.

\section{CONCLUSIONS}

This study has the objective to apply new Internet technologies and multi-agent modelling in order to support the business process of Production Orders Release and especially its capacity planning and control dimension in subcontracting networks. The E-RELEASER is specified as a Web-based Information System (WIS), i.e. an information system (IS) that is based on Web technology and is tightly integrated with conventional IS such as databases and transaction processing systems. The systems design framework of E-RELEASER facilitates internal activities (intranets) and interaction with partners (extranets). Online activities include partner selection, order release and acceptance, and capacity planning and control. Assumed is that the master plan (overall LOB-norm) is fixed by one central point in the manufacturing network, which the prime contractor who has direct contact to the customers. At the moment of an order release to the manufacturing network, there is a contribution to the aggregate released backlog of each partner/work centre that the order will visit. After completion of the manufacturing step, the amount of work (set-up and process time) is deducted from the released backlog of the respective work centre. Every moment we know the released backlog of every partner/work centre, and by controlling the levels of the released backlogs in each partner/work centre, with the predetermined norms of the line-of-balance (B-LOB approach), we ensure that the backlog and the flow of work through the shop is smooth and controlled.

\section{REFERENCES}

[1] BECHTE, W., 1988, Theory and practice of load-oriented manufacturing control, INT.J.PROD.RES., Vol.26, No.3, 375 - 395.

[2] BERTRAND et al., Production Control: A Design-oriented Approach, Elsevier, 1991. 
[3] BOOCH, G. RUMBAUGH, J. and JACOBSON, I., 1999, The unified modelling language user guide, Addison Wesley, Reading.

[4] BROWNE,J. et al,1991, Shop Floor Control Systems, Chapman \& Hall, London.

[5] CHAVEZ, A. and P. MAES, 1996, An agent marketplace for buying and selling goods, Proceedings of the First International Conference on the Practical Application of Intelligent Agents and Mult-Agents Technology (PAAM-96), London, UK, pp. 75 - 90.

[6] COAD, P. and E. YOURDON, 1991, Object-oriented analysis, Prentice-Hall.

[7] DAVIS, M. and D. O'SULLIVAN, 1999, Systems design framework for the extended enterprise, Production Planning \& Control, Vol. 10, No.1, 318.

[8] DAVIS, R. and R.G. SMITH, 1983, Negotiation as a metaphor for distributed problem solving, Artificial Intelligence, vol. 20, pp. $63-100$.

[9] GENESERETH, M.R., 1992, An Agent-based approach to software interoperability, Proceedings of the DAPRA Software technology Conference.

[10] IRASTORZA, J.C., 1974, A loading and balancing methodology for job shop control, AIIE Transactions, Vol. 6, No. 4, pp. 302 - 307.

[11] JENNINGS, N.R. et al, 1998, A roadmap of agent research and development, Autonomous Agents and Multi-Agent Systems 1, 7 - 38, Kluwer, Boston.

[12] JENNINGS, N.R. and W.J. WOOLDRIDGE (Eds), 1998, Agent technology: Foundation, application and markets, Springer Verlag, Berlin.

[13] KINGSMAN, B.G., TATSIOPOULOS, I.P. and HENDRY, L.C., 1989, A structural methodology for managing manufacturing lead times in make-to-order companies, Europ.J.of Oper.Res., 40, 196 - 209.

[14] PARUNAK, H.Van Dyke, 1987, Manufacturing experience with the contract net, in: $\mathbb{M}$. Huhns, editor, Distributed Artificial Intelligence, Pitman, London, pp. 285 - 310.

[15] PETERSEN, S.A., DIVITINI, M. and M. MATSKIN, 2001, An agent-based approach to modelling virual enterprises, Production Planning \& Control, 2001, Vol. 12, No. 3, 224 $-233$.

[16] PLOSSL, G. and WIGHT, O., 1973, Capacity planning and control, Production and Inventory Management, 3rd Qtr., pp. 31 - 67.

[17] SAAD, A., BISWAS, G., KAWAMURA, K., JOHNSON,M.E. and SALAMA, A., 1995, Evaluation of contract net-based hierarchical scheduling for flexible manufacturing systems, Proceedings of intelligent Manufacturing Workshop at IJCAI95, Montreal, pp.310 - 321.

[18] SADEH, N.M. et al, MASCOT, 2001, An agent-based architecture for dynamic supply chain creation and coordination in the internet economy, Production Planning and Control, 2001, Vol. 12, No. 3, $212-223$.

[19] SADEH, N. and FOX, M.S, 1989, CORTES: An exploration into micro-opportunistic job-shop scheduling, Proceedings of Workshop on Manufacturing Production Scheduling at IJCAI-89, Detroit.

[20] SANDHOLM, Th., 1997, An implementation of the contract net protocol based on marginal cost calculations, Proceedings of the First International Conference on Autonomous Agents (Agents 97), Marinal del Rey, CA, pp. 209 - 216.

[21] SHEN, W and NORRIE, D.H, 1999, Agent-based systems for intelligent manufacturing: A state-of-the-art survey, International Journal for Knowledge and Information Systems, 1(2), $129-156$.

[22] TANENBAUM. A.S. and M. van STEEN, 2002, Distributed systems: Principles and paradigms, Prentice Hall, New Jersey.

[23] TATSIOPOULOS, I.P., 1997, An Orders release reference model as a link between production management and shop floor control software, Computers in Industry 33,pp. $335-344$.

[24] WANG, S., 2001, Toward a general model for web-based information systems, Int. J. of Information Management, 21, 385 - 396. 
[25] WIENDHAL, H.P., 1987, Belastungsorientierte Fertigungssteuerung, (Muenchen: Carl Hanser Verlag).

[26] WORTMANN, H. and N. SZIRBIK, 2001, ICT issues among collaborative enterprises: from rigid to adaptive agent-based technologies, Production Planning \& Control, Vol. $12,452-465$. 